\title{
Effects of macroscopic propagation on spectra of broadband supercontinuum harmonics and isolated-attosecond-pulse generation: Coherent control of the electron quantum trajectories in two-color laser fields
}

\author{
Peng-Cheng $\mathrm{Li}^{1,2, *}$ and Shih-I Chu ${ }^{3, \dagger}$ \\ ${ }^{1}$ Center for Quantum Science and Engineering, Department of Physics, National Taiwan University, Taipei 10617, Taiwan \\ ${ }^{2}$ College of Physics and Electronic Engineering, Northwest Normal University, Lanzhou 730070, China \\ ${ }^{3}$ Department of Chemistry, University of Kansas, Lawrence, Kansas 66045, USA
}

(Received 10 May 2012; published 13 July 2012)

\begin{abstract}
Recently it was shown that broadband supercontinuum harmonics can be produced from the long-trajectory electrons in the single-atom response by the coherent control of the electron trajectories through optimized two-color laser fields. Such supercontinuum harmonics can be superposed to generate an isolated sub-30attosecond (as) pulse [Liu et al., Phys. Rev. A 84, 033414 (2011)]. In this paper, we investigate the effect of macroscopic propagation on the supercontinuum harmonic spectra and the subsequent attosecond-pulse generation of atomic hydrogen. The time-dependent Schrödinger equation is solved accurately and efficiently by means of the time-dependent generalized pseudospectral method. The effects of macroscopic propagation are investigated in near and far field by solving Maxwell's equation. The results show that the contribution of short-trajectory electron emission is increased when the macroscopic propagation is considered. However, the characteristics of the dominant long-trajectory electron emission (in the single-atom response case) are not changed, and an isolated 53 as pulse can be generated in the near field. Moreover, in the far field, the contribution of long-trajectory electron emission is still dominant for both on-axis and off-axis cases. As a result, an isolated 42 as pulse can be generated directly. Similar results are obtained when the atomic target position is changed. Therefore, the proposed method for the single ultrashort attosecond-pulse generation can be realized by means of the coherent control of the electron quantum paths in appropriately optimized two-color laser fields.
\end{abstract}

DOI: 10.1103/PhysRevA.86.013411

PACS number(s): $32.80 . \mathrm{Rm}, 42.50 . \mathrm{Hz}, 42.65 . \mathrm{Ky}$

\section{INTRODUCTION}

In the last decade, attosecond (as) science and ultrafast technology has grown quickly into one of the most exciting scientific new frontiers in the 21 st century [1-4]. In particular, it has been experimentally demonstrated that single and multiple attosecond laser pulses can be produced by means of the process of high-order harmonic generation (HHG) in rare gases [5,6]. As the length of the incident pulse decreases, the HHG peaks in the cutoff regime are replaced by one or a few pronounced supercontinuum patterns as individual adjacent peaks begin to merge with one another [7,8]. For few-cycle driving pulses, it is found that the emission of the consecutive harmonics in the supercontinuum cutoff regime can be synchronized and locked in phase, resulting in the production of coherent attosecond pulses [8]. Moreover, the time-frequency profile of the attosecond laser pulses can be controlled by tuning the carrier envelope phase (CEP) [8].

Today isolated ultrashort XUV pulses with pulse lengths of the order of 100 as are produced in several laboratories worldwide, leading to a number of exciting new advancements in atomic, molecular, and optical physics and other scientific subfields. Attosecond laser pulse can now manipulate basic ultrafast electronic processes, such as observation of control of electron wave packets [9], probing of nuclear dynamics [10] and electronic dynamics [11], attosecond time-resolved

\footnotetext{
*lipc@nwnu.edu.cn

${ }^{\dagger}$ sichu@ku.edu
}

spectroscopy [12], tomographic imaging of molecular orbitals [13], etc.

Currently, the production of single attosecond pulses can be achieved experimentally [6] by means of the spectral selection of HHG broadband supercontinuum. Several methods have been proposed for the generation of the supercontinuum harmonics spectra and the generation of single attosecond pulses, such as using a few-cycle laser pulse [8], double optical gating [14], the chirped laser pulse [15], the multicycle driving pulses [16], and so on. In addition, several alternative methods have been suggested by using the two- or multicolor laser scheme for generating an intense single attosecond pulse [17-19]. In particular, coherent control of the electron quantum paths is an efficient method for the production of the broadband supercontinuum harmonics spectra and isolated attosecond pulse [20-23]. It is known that there are two types of dominant quantum trajectories (the so-called short and long trajectory) that contribute to each harmonic emission. As the two quantum trajectories have different ionizing and returning times, their harmonic phases often behave differently. As a result, the synthesis of several harmonics often leads to an irregular attosecond pulse. In order to obtain a single and coherent attosecond pulse, it is advantageous to select only one type of the two trajectories by coherent control of the electron quantum paths. Normally, only the HHG spectra from the short trajectories are observed on axis in the gas target experimentally [24]. In other words, the harmonics spectra from long trajectories are usually not dominant on axis after considering macroscopic propagation. However, from our recent study [25], it is found that ultrabroadband supercontinuum spectra, and ultrashort (sub-30 as) single attosecond pulses can 
be generated from the dominant long-trajectories harmonics alone by means of the coherent control of the electron quantum trajectories in the single-atom response using appropriately optimized two-color laser pulses. But an important question remains to be explored, namely, the effect of macroscopic propagation. Will the broadband supercontinuum spectra from the dominant long-trajectories electrons in the single-atom response case still be maintained on axis or off axis after the macroscopic propagation? This issue must be investigated before the single ultrashort attosecond-pulse generation can be realized by means of the metrology of optimizing the incident laser pulse shape and coherent control of the electron quantum trajectories using two-color [25] or three-color [26] laser pulses.

In this paper, we investigate the effect of macroscopic propagation on supercontinuum spectra produced by the coherent control of the electron quantum trajectories using the optimized two-color laser field. The propagation is performed by solving the three-dimensional (3D) Maxwell equation. The time-dependent Schrödinger equation (TDSE) is solved by means of the time-dependent generalized pseudospectral method (TDGPS) [27]. The TDGPS method allows nonuniform and optimal spatial discretization and accurate and efficient time propagation of the wave function with the use of only a modest number of spatial grid points. This greatly facilitates the accurate treatment of the macroscopic propagation effects for HHG. In this paper, we will investigate the effect of the macroscopic propagation on the supercontinuum harmonic spectra obtained from the single-atom response, as well as its effects on the ultrashort attosecond-pulse generation by selecting the dominant long trajectory in the macroscopic level.

This paper is organized as follows. In Sec. II, we briefly describe the theoretical and computational methods about single-atom response and macroscopic propagation. In Sec. III, we discuss the effects of macroscopic propagation on the supercontinuum spectra and on the single ultrashort attosecondpulse generation from the dominant long trajectories by means of the optimized two-color field in near field and in far field. This is followed by a conclusion in Sec. IV.

\section{THEORETICAL METHODS}

HHG produced by the interaction of an intense laser field with a single atom can be calculated by solving the TDSE, in atomic units,

$$
i \frac{\partial \psi(\mathbf{r}, t)}{\partial t}=\hat{H} \psi(\mathbf{r}, t)=\left[\hat{H}_{0}+\hat{V}(\mathbf{r}, t)\right] \psi(\mathbf{r}, t) .
$$

Here $\hat{H}_{0}$ represents unperturbed hydrogen atom Hamiltonian and $\hat{V}(\mathbf{r}, t)$ is the time-dependent atom-field interaction given by

$$
\hat{V}(\mathbf{r}, t)=-\mathbf{E}(t) \cdot \mathbf{r}=-z E(t),
$$

where $E(t)$ is the electric field. The TDSE can be solved accurately and efficiently by means of the TDGPS method [27]. The numerical scheme of the TDGPS method consists of two essential steps: (i) The spatial coordinates are optimally discretized in a nonuniform fashion by means of the generalized pseudospectral (GPS) technique: denser grids near the nuclear origin and sparser grids for larger distances. (ii) A second-order split-operator technique in the energy representation, which allows the explicit elimination of undesirable fast-oscillating high-energy components, is used for the efficient and accurate time propagation of the wave function:

$$
\begin{aligned}
\psi(\mathbf{r}, t+\Delta t) \simeq & \exp \left(-i \hat{H}_{0} \frac{\Delta t}{2}\right) \exp \left[-i \hat{V}\left(\mathbf{r}, \theta, t+\frac{\Delta t}{2}\right) \Delta t\right] \\
& \times \exp \left(-i \hat{H}_{0} \frac{\Delta t}{2}\right) \psi(\mathbf{r}, t)+O\left(\Delta t^{3}\right) .
\end{aligned}
$$

The TDGPS technique is considerably more efficient and accurate than the conventional time-dependent technique using equal-spacing grid discretization. The unitarity of the wave function is automatically preserved by this procedure and the norm of the field-free wave function is preserved to at least ten digits of accuracy during the time propagation. Once the timedependent wave function $\psi(\mathbf{r}, t)$ is obtained, we can calculate the expectation value of the induced dipole acceleration,

$$
\begin{aligned}
d_{A}(t) & =\left\langle\psi(\mathbf{r}, t)\left|\frac{d^{2} z}{d t^{2}}\right| \psi(\mathbf{r}, t)\right\rangle \\
& =\left\langle\psi(\mathbf{r}, t)\left|-\frac{z}{\mathbf{r}^{3}}+E(t)\right| \psi(\mathbf{r}, t)\right\rangle .
\end{aligned}
$$

The HHG spectrum in the single-atom level can be obtained by the Fourier transformation of time-dependent dipole acceleration as follows:

$$
P_{S}(\omega)=\left|\frac{1}{t_{f}-t_{i}} \frac{1}{\omega^{2}} \int_{t_{i}}^{t_{f}} d_{A}(t) e^{-i \omega t}\right|^{2} .
$$

The harmonic field propagation in a macroscopic medium is described by a 3D Maxwell equation [28-31]

$$
\nabla^{2} E_{h}\left(r^{\prime}, z^{\prime}, t\right)-\frac{1}{c^{2}} \frac{\partial^{2} E_{h}\left(r^{\prime}, z^{\prime}, t\right)}{\partial t^{2}}=\mu_{0} \frac{\partial^{2} D\left(r^{\prime}, z^{\prime}, t\right)}{\partial t^{2}},
$$

where

$$
D\left(r^{\prime}, z^{\prime}, t\right)=\left[n_{0}-n_{e}\left(r^{\prime}, z^{\prime}, t\right)\right] d_{A}\left(r^{\prime}, z^{\prime}, t\right),
$$

$n_{0}$ is the neutral atom density, $n_{e}\left(r^{\prime}, z^{\prime}, t\right)$ is free-electron density, and $d_{A}\left(r^{\prime}, z^{\prime}, t\right)$ is the single-atom induced dipole acceleration. Equation (6) is solved in the frequency domain. Once the harmonic field is obtained at the exit face, the HHG spectrum in macroscopic level can be obtained by integration over the transverse direction,

$$
P_{M}(\omega) \propto \int_{0}^{\infty} 2 \pi r^{\prime} d r^{\prime}\left|E_{h}\left(r^{\prime}, \omega\right)\right|^{2},
$$

and the intensity of attosecond pulses can be calculated as follows [32]:

$$
I(t)=\int_{0}^{\infty} 2 \pi r^{\prime} d r^{\prime}\left|\int_{\omega_{1}}^{\omega_{2}} E_{h}\left(r^{\prime}, \omega\right) e^{i \omega t} d \omega\right|^{2} .
$$

To explore the detailed spectral and temporal timedependent structures of $\mathrm{HHG}$, we perform a time-frequency analysis by means of the wavelet transform of the harmonic field $E_{h}\left(t^{\prime}\right)$ [33],

$$
A(t, \omega)=\int E_{h}\left(t^{\prime}\right) \sqrt{\omega} W\left[\omega\left(t^{\prime}-t\right)\right] d t^{\prime} .
$$

Here, $\omega$ is the fundamental laser frequency and $W\left[\omega\left(t^{\prime}-t\right)\right]$ is the mother wavelet. For the harmonic emission, a natural 

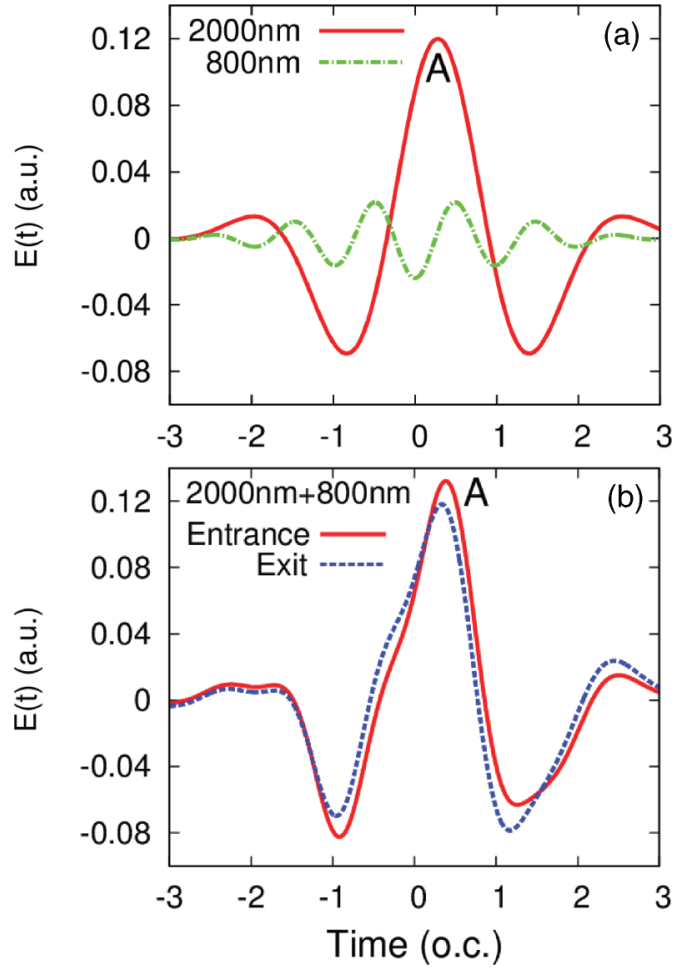

FIG. 1. (Color online) (a) A one-color $800 \mathrm{~nm}$ laser pulse and a one-color $2000 \mathrm{~nm}$ laser pulse with proper time delay, the peak intensity $I_{1}=2.0 \times 10^{13} \mathrm{~W} / \mathrm{cm}^{2}$ and $I_{2}=5.0 \times 10^{14} \mathrm{~W} / \mathrm{cm}^{2}$, respectively; the duration of each pulse is 5 fs (FWHM). (b) The optimized two-color laser field at the entrance and at the exit (on axis).

choice of mother wavelet is given by the Morlet wavelet,

$$
W(x)=\frac{1}{\sqrt{\tau}} e^{i x} e^{-x^{2} / 2 \tau^{2}} .
$$

\section{RESULTS AND DISCUSSION}

In our simulation, the optimized two-color electric field has the following form:

$$
\begin{aligned}
E\left(r^{\prime}, z^{\prime}, t\right)= & \operatorname{Re}\left\{E_{1}\left(r^{\prime}, z^{\prime}\right) f_{1}\left(r^{\prime}, z^{\prime}, t\right) e^{-i\left(\omega_{1} t+\varphi_{1}\right)}\right. \\
& \left.+E_{2}\left(r^{\prime}, z^{\prime}\right) f_{2}\left(r^{\prime}, z^{\prime}, t-t_{d}\right) e^{-i\left[\omega_{2}\left(t-t_{d}\right)+\varphi_{2}\right]}\right\},
\end{aligned}
$$

where $E_{1}\left(r^{\prime}, z^{\prime}\right)$ and $E_{2}\left(r^{\prime}, z^{\prime}\right)$ are the Gaussian beams with the laser duration $f_{1}\left(r^{\prime}, z^{\prime}, t\right)$ and $f_{2}\left(r^{\prime}, z^{\prime}, t\right)$, respectively, and $\omega_{1}$ and $\omega_{2}$ are the corresponding frequencies. $\varphi_{1}$ and $\varphi_{2}$ are the carrier envelope phases for each Gaussian beam, and $t_{d}$ is the time delay between the two laser pulses. Figure 1(a) shows a one-color $800 \mathrm{~nm}$ laser pulse and a one-color $2000 \mathrm{~nm}$ laser pulse with proper time delay; the maximum peak is marked by $A$. The peak intensity is $I_{1}=2.0 \times 10^{13} \mathrm{~W} / \mathrm{cm}^{2}$ and $I_{2}=5.0 \times 10^{14} \mathrm{~W} / \mathrm{cm}^{2}$, respectively, and the duration of each Gaussian beam is 5 fs (full width at half maximum [FWHM]). The time delay is $t_{d}=0.28 T_{1}\left(T_{1}=2 \pi / \omega_{1}\right)$, and the carrier envelope phases are $\varphi_{1}=\pi$ and $\varphi_{2}=0$, respectively. Figure 1(b) shows the on-axis optimized two-color laser field at the entrance (solid line) and at the exit (dotted line), assuming that the optimized two-color fundamental electric field has the same laser focus, and the laser field propagates

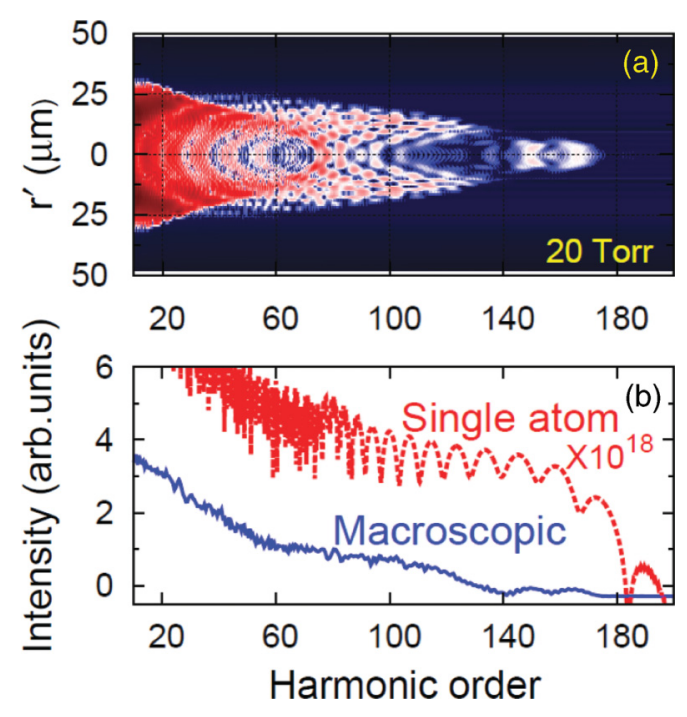

FIG. 2. (Color online) (a) Spatial distribution of macroscopic HHG spectra from hydrogen atoms at the exit face. The length of the atomic target is $0.2 \mathrm{~mm}$, the pressure is 20 Torr, and the target is set at the laser focus $\left(z^{\prime}=0 \mathrm{~mm}\right)$. (b) Comparison of the HHG for the single-atom response and the macroscopic case. The laser parameters used are the same as those in Fig. 1.

in the free space, and the maximum peak is also marked by $A$. Such optimized two-color laser fields can be obtained by the combination of a one-color $800 \mathrm{~nm}$ laser pulse and a one-color $2000 \mathrm{~nm}$ laser pulse with proper time delay, and the maximum peak $A$ is right shifted. According to our previous work [25], the right-shifted case is better for the generation of the broadband supercontinuum spectrum and isolated attosecond pulse. On the other hand, the peak intensity of the optimized two-color laser field at the exit is smaller than that at the entrance, and the shape of the exit pulse is changed in comparison with the entrance pulse case. The reason is that the intensity and geometric phase of the two laser pulses are different at the entrance face and exit face, so the shape of the combination of the two laser pulses is also different.

In Fig. 2(a), we present the spatial distribution of macroscopic HHG from hydrogen atoms at the exit face, where the atomic target is put at $z^{\prime}=0 \mathrm{~mm}$, the length of the target is $0.2 \mathrm{~mm}$, the pressure used is 20 Torr, the beam waist at the laser focus is $25 \mu \mathrm{m}$, and all the other laser parameters are the same as those in Fig. 1. We can see that the intensity of harmonic emission is different in the spatial distribution, reflecting the complex phase changes in the macroscopic level. In order to demonstrate the generation of the supercontinuum spectra by the optimized two-color laser field in the singleatom response, and the effects of macroscopic propagation on supercontinuum spectra, we show the single-atom and macroscopic HHG spectra from hydrogen atoms in Figs. 2(a) and 2(b), respectively. For the single-atom response, the cutoff of the HHG is located near the 180th order, and there is a broader and smoother supercontinuum plateau from the 95 th to 180th orders. For the macroscopic case, the cutoff of the HHG is smaller than the single-atom case, and the supercontinuum plateau has a shift toward the lower harmonic order.

To figure out the detailed spectral and temporal timedependent structures of $\mathrm{HHG}$ for a single-atom response 


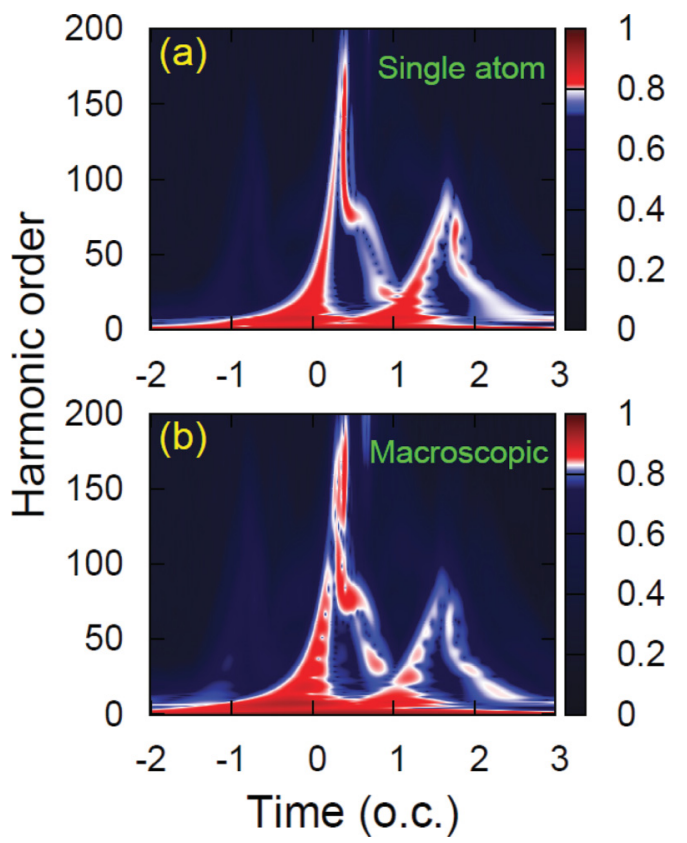

FIG. 3. (Color online) Wavelet time-frequency analysis of the HHG spectra. (a) Single-atom response. (b) Macroscopic case, on axis. The laser parameters used are the same as those in Fig. 1.

and macroscopic case, we perform a time-frequency analysis of the HHG by means of the wavelet transform, which is shown in Figs. 3(a) and 3(b), respectively. For the single-atom response, there is one major emission burst, which comes from the contribution of the long trajectory, and an extreme broadband supercontinuum spectrum can be obtained from the 95th to 180th orders. This broadband supercontinuum spectrum is produced by optimizing the two-color laser field. As shown before [25], the optimized two-color laser field allows long-trajectory electrons to be emitted in a very narrow time range and contribute to each harmonic order near the cutoff region. However, the short-trajectory electrons emit in a longer time range. In other words, this optimized two-color laser field not only prolongs the short-trajectory electron emission time and suppresses the short-trajectory contribution, but also stimulates abundant long-trajectory electrons to emit harmonic radiation within a short time span. For the macroscopic case [see Fig. 3(b)], we find that the major emission burst still comes from the contribution of the long trajectory. But, the trajectory is broken near the 120th order, namely, the supercontinuum plateau intensity is decreased near that region. In fact, we can see from the spatial distribution of macroscopic harmonic spectra shown in Fig. 2(a) that the intensity of on-axis harmonic spectra is very low from the 100 th order to 140th order, but the corresponding off-axis case is higher. Therefore, we integrated over the transverse direction to the calculation of macroscopic harmonic spectra; the broken trajectory near the 120th order leaves no trace in the macroscopic case as shown in Fig. 2(b). On the other hand, the contribution of the short-trajectory electrons in the broadband supercontinuum region are somewhat enhanced after the macroscopic propagation.

It is known that the HHG phase behavior is very important for the generation of broadband supercontinuum spectra and
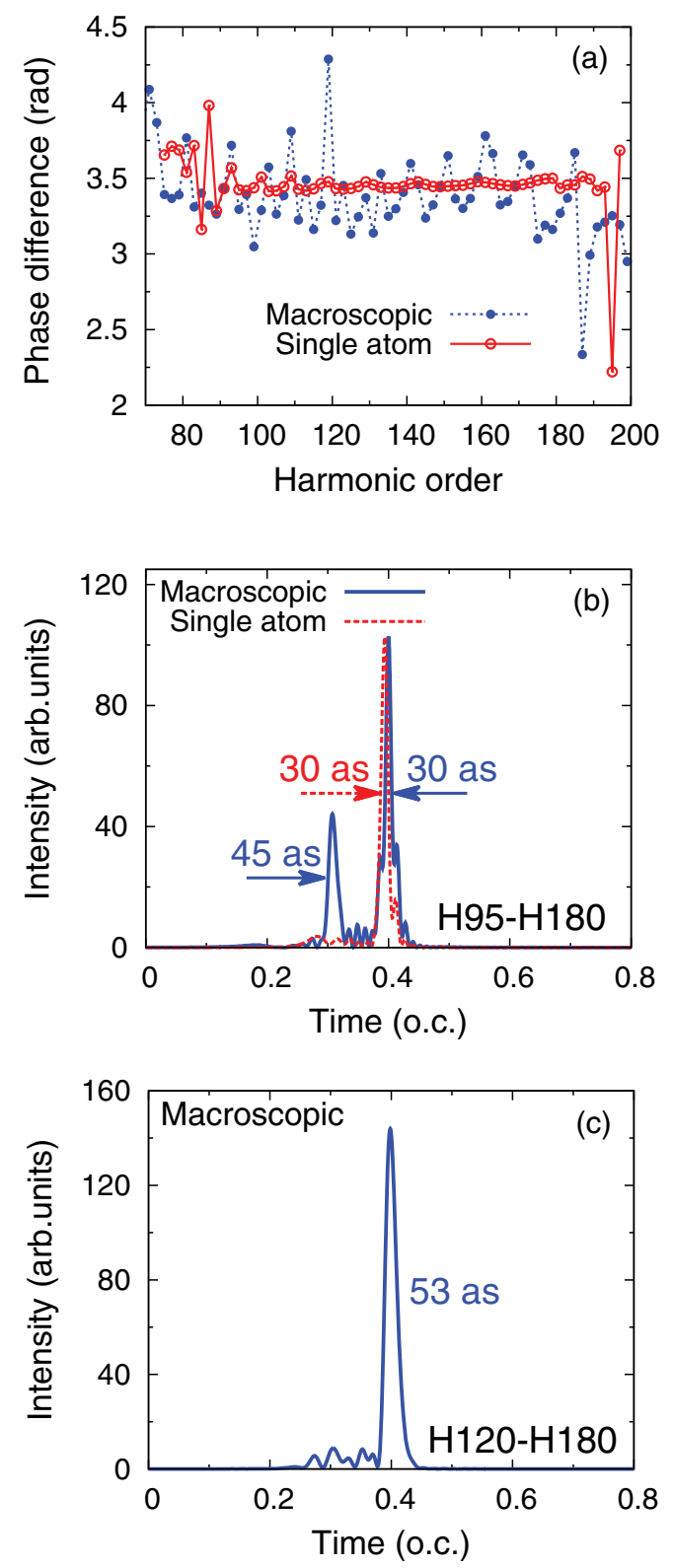

FIG. 4. (Color online) (a) Comparison of phase difference from the single-atom response and the macroscopic case. (b) The corresponding attosecond pulses obtained by superposing the harmonics from the 95th to 180th order (H95-H180). For comparison, the intensity of the macroscopic attosecond pulses is divided by a constant. (c) Macroscopic attosecond pulses by superposing the harmonics from the 120th to 180th order (H120-H180). The laser parameters used are the same as those in Fig. 1.

attosecond pulse. The phase locking does not mean that the harmonic components have the same phase, but rather that the phase difference between two consecutive harmonics is constant. Figure 4(a) shows the phase difference dependence on the harmonic order for the single-atom response and macroscopic cases, respectively. We can see that the phase difference is almost a constant for single-atom response from the 95 th to 180 th order. It means that the harmonic phase is nearly locked, and the superposition of these harmonics leads to the regular attosecond pulse shown in Fig. 4(b). 
For the macroscopic case, the phase difference is apparently changed, and the phase lock is not as good as the single-atom response. Note that near the 120th order, there is a sharp change of the phase difference. Therefore, the harmonics in this region are not phase locked, so the intensity of harmonic emission is decreased suddenly. Thus the harmonic spectra near the 120th order leaves no trace in the macroscopic case shown in Fig. 3(b). In Fig. 4(b), we compare the generation of the attosecond pulse for the single-atom response and macroscopic case, which are obtained by superposing 85 orders of harmonics from the 95th to the 180th order. An isolated 30 as pulse is obtained directly from the dominant long trajectory for the single-atom response case. For the macroscopic case, we also obtain an isolated 30 as pulse, and a weaker 45 as pulse due to the contribution of short trajectory after propagation. However, an isolated 53 as pulse can still be obtained in macroscopic level when only the harmonics from the 120th to 180th orders are selected, as shown in Fig. 4(c). Therefore, this result demonstrates that our proposed method using optimized two-color laser fields is achievable to the macroscopic case.

We know that the contribution of the short and long trajectory to harmonic emission is different in spatial distribution. Particularly in the far field, the short and long trajectory are spatially separated. Normally, the contribution of short trajectory is dominant on axis [24], and the contribution of long trajectory can also be observed off axis at large divergence region. In the following, we will discuss the HHG spectra and attosecond-pulse generation in the far field, which are produced by the dominant long trajectory. Note that the far-field harmonic emissions can be obtained by the Hankel

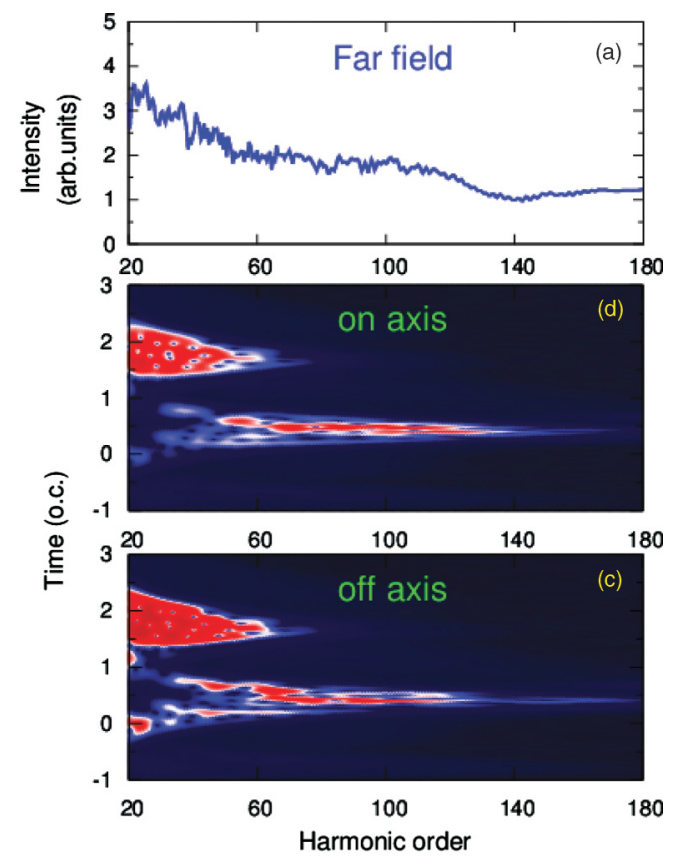

FIG. 5. (Color online) (a) Macroscopic HHG spectra in far field $\left(z^{\prime \prime}=30 \mathrm{~cm}\right)$; the corresponding wavelet time-frequency spectra for the on-axis $\left(r^{\prime \prime}=0 \mathrm{~mm}\right)$ and off-axis $\left(r^{\prime \prime}=0.2 \mathrm{~mm}\right)$ cases are shown in (b) and (c), respectively. The laser parameters used are the same as those in Fig. 1. transformation of the near-field harmonic emissions [34,35]:

$$
\begin{aligned}
E_{h}\left(r^{\prime \prime}, z^{\prime \prime}, \omega\right)= & -i k \int \frac{E_{h}\left(r^{\prime}, z^{\prime}, \omega\right)}{z^{\prime \prime}-z^{\prime}} J_{0}\left(\frac{k r^{\prime} r^{\prime \prime}}{z^{\prime \prime}-z^{\prime}}\right) \\
& \times \exp \left[\frac{i k\left(r^{\prime 2}+r^{\prime 2}\right)}{2\left(z^{\prime \prime}-z^{\prime}\right)}\right] r^{\prime} d r^{\prime},
\end{aligned}
$$

where $J_{0}$ is the zero-order Bessel function and $z^{\prime \prime}$ is the far-field position from the laser focus. In Fig. 5(a), we show the macroscopic HHG spectra in far field $\left(z^{\prime \prime}=30 \mathrm{~cm}\right)$. Note that the calculation of far-field HHG spectra involves the integration over the radial coordinate. We can see that the cutoff of the HHG is located near the 160th order, which is smaller than that of the near-field case. In Fig. 5(b), we show the corresponding wavelet time-frequency analysis in far field for both the on-axis $\left(r^{\prime \prime}=0 \mathrm{~mm}\right)$ and off-axis $\left(r^{\prime \prime}=0.2 \mathrm{~mm}\right)$ cases, respectively. For the on-axis case, we can see that there is a major emission burst, and the contribution of the long trajectory is dominant. The contribution of the short trajectory is also increased, compared with the single-atom response. However, for the off-axis case, only the long quantum trajectory is dominant, as shown in Fig. 5(c). We can see that the harmonic emission from the 80th to 150th takes place at the same time. This is advantageous to the generation of ultrashort single attosecond pulses.
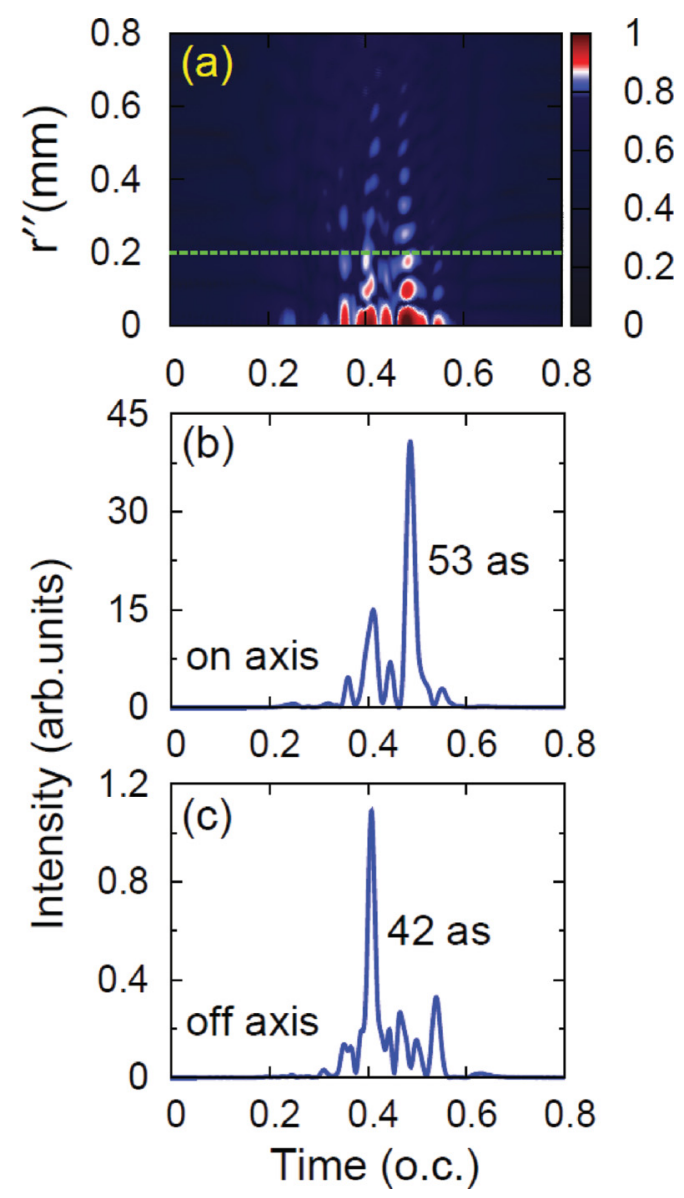

FIG. 6. (Color online) Attosecond-pulse generation in the far field. (a) Spatial distribution; (b) on axis $\left(r^{\prime \prime}=0 \mathrm{~mm}\right)$; and (c) off axis $\left[r^{\prime \prime}=0.2 \mathrm{~mm}\right.$ shown by the green solid line in (a)]. The laser parameters used are the same as those in Fig. 1. 


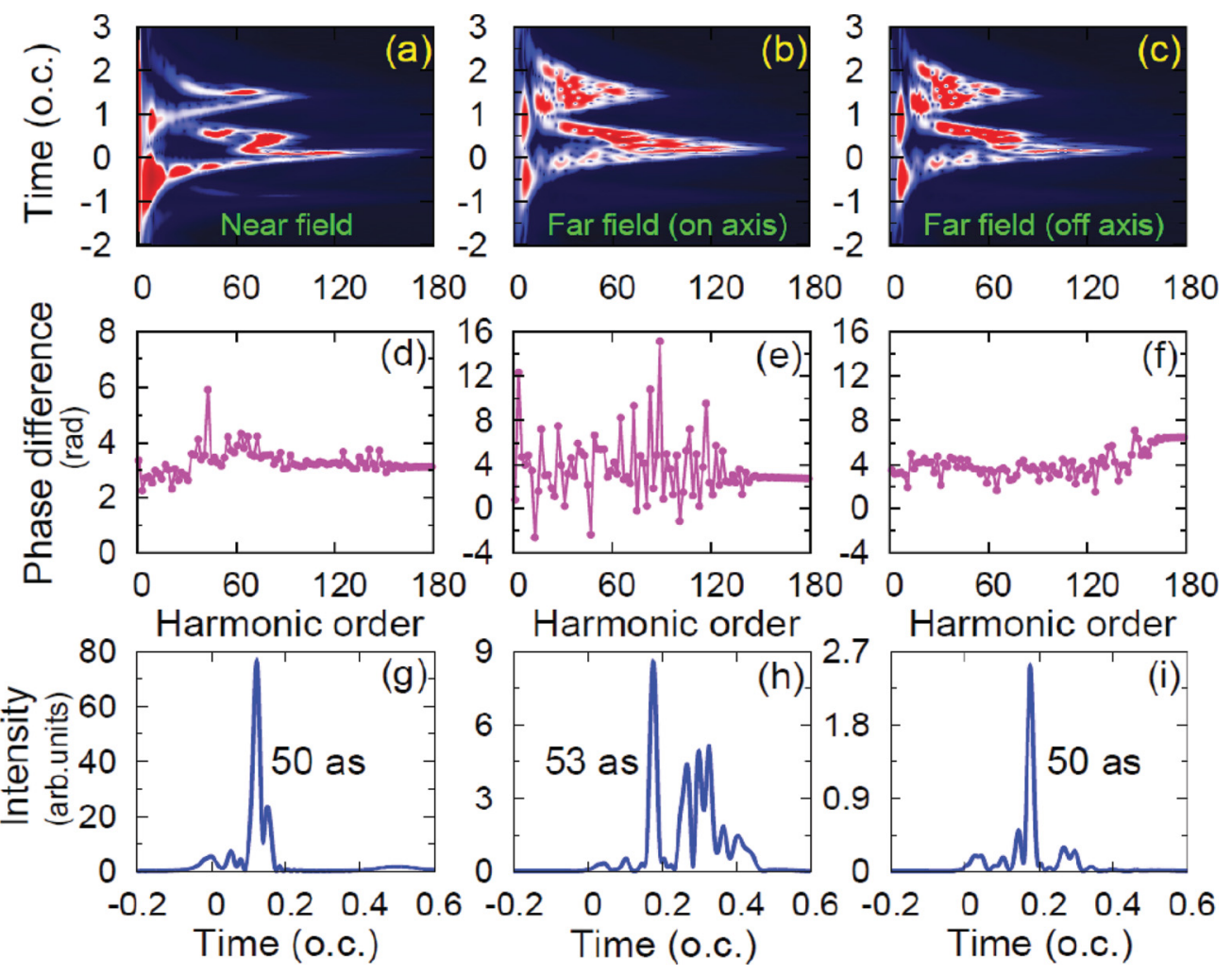

FIG. 7. (Color online) Wavelet time-frequency analysis of harmonic spectra for (a) the on-axis near field, (b) on-axis far field, and (c) off-axis far field. Note that the off axis is located at $r^{\prime \prime}=0.1 \mathrm{~mm}$, and the atomic target is set at $0.5 \mathrm{~mm}$ after the laser focus. The corresponding phase differences are shown in (d), (e), and (f), respectively. (g) Attosecond-pulse generation from the on-axis near field by superposing the harmonics from the 85th to 140th order. (h) and (i) are the on-axis and off-axis far-field cases by superposing the harmonics from the 80th to 130th order. The other parameters are the same as those in Fig. 1.

In Fig. 6(a), we present the intensity distribution of the synthesized harmonic spectra from the 80th to 150th order in space. We can see that the contribution of the short trajectory is decreased at off-axis far field, but the long trajectories there are the distribution far from the propagation axis. In Figs. 6(b) and $6(\mathrm{c})$, we present the far-field attosecondpulse generation for the on-axis and off-axis cases shown in Figs. 5(b) and 5(c), respectively. We see that a single 53 as pulse is obtained from the on-axis far-field harmonic spectra, which is the same as that in the near-field case, and another single 42 as pulse is obtained from the off-axis far-field harmonic spectra.

In order to demonstrate the effect of the position of the atomic target on the broadband supercontinuum spectra, we now move the atomic target to $0.5 \mathrm{~mm}$ after the laser focus, and keep the other parameters the same as the previous case. In Fig. 7(a), we present the wavelet time-frequency analysis of harmonic spectra for the on-axis near-field case. It shows only one major emission burst, which comes from the contribution of the long quantum trajectory. Furthermore, we find that the HHG cutoff is located near the 160th order. It is smaller than the previous cases [see Fig. 2(b) or Fig. 3(b)] because the intensity of the laser field becomes smaller after the laser focus. In Fig. 7(d), the corresponding phase difference from consecutive harmonic spectra is shown, indicating good phase lock from the 85th to 140th order, since the phase difference is almost a constant. This result provides a good explanation to the behavior of those harmonics which exhibit the same emission time as shown in Fig. 7(a). By superposing the given range of harmonics, we obtain a single 50 as pulse from on-axis near field, as shown in Fig. 7(g).

In Fig. 7(b), we present the wavelet time-frequency analysis of harmonic spectra from the on-axis $\left(r^{\prime \prime}=0 \mathrm{~mm}\right)$ far-field case. It shows that the contribution of short trajectories is increased, which is originally quite weak for the single-atom response. This result can be understood easily from the corresponding phase difference, as shown in Fig. 7(e). We can see that the phase difference is changed rapidly from the 80th to 130th order, and the phase lock of the HHG spectrum is not good. Note that the long trajectory is still dominant [see Fig. 7(b)], although the contribution of short trajectory is increased in this case. The synchronization of harmonics is also partly destroyed. Therefore, although we can still obtain a single 53 as pulse from on-axis far field, as shown in Fig. 7(h), several satellite peaks are also produced.

In Fig. 7(c), we present the wavelet time-frequency analysis of harmonic spectra for the off-axis far-field case. The of axis is located at $r^{\prime \prime}=0.1 \mathrm{~mm}$. It shows that the long trajectory is dominant from the 80th to 130th order, and all the harmonics emit at about the same time. Furthermore, the corresponding phase difference is locked at a relatively smaller constant, as shown in Fig. 7(f). This implies that there is a good synchronization for the given range of harmonics. As a result, a clean and isolated 50 as pulse is generated directly, as shown in Fig. 7(i). We can see that this single attosecond pulse is 
regular and smooth, which only comes from the dominant long trajectory, thanks to the large divergence in off-axis far field. This result also agrees with the earlier prediction that only one quantum trajectory is observed experimentally in the far field [24].

\section{CONCLUSIONS}

In this paper, we investigate the effect of macroscopic propagation on the broadband supercontinuum spectrum of atomic hydrogen produced by the coherent control of quantum trajectories using an optimized two-color laser field. In the optimized two-color laser field, only long-trajectory electrons are dominant near the cutoff region, and the HHG spectra are emitted in a very short time range, leading to a broadband supercontinuum. The superposition of the supercontinuum harmonics yields a coherent ultrashort 30 as pulse in the single-atom response case. After considering the macroscopic propagation, we find that a single 53 as pulse can still be generated from the dominant long-trajectory electrons in the near field. For the on-axis far-field case, we obtain similar results. For the off-axis far-field case, because of the large divergence, only the harmonic emission from the long-trajectory electrons continues to prevail, and the emission from the short-trajectory electrons becomes very weak. This is advantageous to the generation of single attosecond pulses. As a result, an ultrashort 42 as pulse can be generated directly. Finally, we also study the dependence of the broadband supercontinuum spectrum on the atomic target position, and find that the attosecond-pulse generation is not sensitive to the atomic target position. In conclusion, the proposed method in this paper is efficient and can be realized for the generation of isolated ultrashort attosecond pulse by selecting the dominant long-trajectory emission using the optimized two-color laser field.

\section{ACKNOWLEDGMENTS}

This work was partially supported by the US Department of Energy. P.-C.L. is partially supported by the National Natural Science Foundation of China (Grant No. 11044007 and No. 11047016). We also would like to acknowledge the partial support of National Science Council and National Taiwan University.
[1] M. Hentschel, R. Kienberger, Ch. Spielmann, G. A. Reider, N. Milosevic, T. Brabec, P. Corkum, U. Heinzmann, M. Drescher, and F. Krausz, Nature (London) 414, 509 (2001).

[2] P. M. Paul, E. S. Toma, P. Breger, G. Mullot, F. Augé, Ph. Balcou, H. G. Muller, and P. Agostini, Science 292, 1689 (2001).

[3] F. Krausz and M. Ivanov, Rev. Mod. Phys. 81, 163 (2009).

[4] Z. H. Chang and P. Corkum, J. Opt. Soc. Am. B 27, B9 (2010).

[5] J. Mauritsson, P. Johnsson, E. Gustafsson, A. L'Huillier, K. J. Schafer, and M. B. Gaarde, Phys. Rev. Lett. 97, 013001 (2006).

[6] E. Goulielmakis et al., Science 320, 1614 (2008).

[7] R. Kienberger et al., Nature (London) 427, 817 (2004).

[8] J. J. Carrera, X. M. Tong, and Shih-I. Chu, Phys. Rev. A 74, 023404 (2006).

[9] R. Kienberger et al., Science 297, 1144 (2002).

[10] S. Baker, J. S. Robinson, C. A. Haworth, H. Teng, R. A. Smith, C. C. Chirilă, M. Lein, J. W. G. Tisch, and J. P. Marangos, Science 312, 424 (2006).

[11] M. Uiberacker et al., Nature (London) 446, 627 (2007).

[12] M. Drescher, M. Hentschel, R. Kienberger, M. Uiberacker, V. Yakovlev, A. Scrinzi, Th. Westerwalbesloh, U. Kleineberg, U. Heinzmann, and F. Krausz, Nature (London) 419, 803 (2002).

[13] J. Itatani, J. Levesque, D. Zeidler, H. Niikura, H. Pépin, J. C. Kieffer, P. B. Corkum, and D. M. Villeneuve, Nature (London) 432, 867 (2004).

[14] Z. H. Chang, Phys. Rev. A 76, 051403(R) (2007).

[15] J. J. Carrera and Shih-I. Chu, Phys. Rev. A 75, 033807 (2007).

[16] I. Thomann, A. Bahabad, X. Liu, R. Trebino, M. M. Murnane, and H. C. Kapteyn, Opt. Express 17, 4611 (2009).

[17] E. J. Takahashi, P. Lan, O. D. Muecke, Y. Nabekawa, and K. Midorikawa, Phys. Rev. Lett. 104, 233901 (2010).
[18] Y. Oishi, M. Kaku, A. Suda, F. Kannari, and K. Midorikawa, Opt. Express 14, 7230 (2006).

[19] Z. Zeng, Y. Cheng, X. Song, R. Li, and Z. Xu, Phys. Rev. Lett. 98, 203901 (2007).

[20] G. Sansone et al., Science 314, 443 (2006).

[21] K. J. Schafer, M. B. Gaarde, A. Heinrich, J. Biegert, and U. Keller, Phys. Rev. Lett. 92, 023003 (2004).

[22] M. Fieß, B. Horvath, T. Wittmann, W. Helml, Y. Cheng, B. Zeng, Z. Xu, A. Scrinzi, J. Gagnon, F. Krausz, and R. Kienberger, New J. Phys. 13, 033031 (2011).

[23] W. Boutu et al., Nat. Phys. 4, 545 (2008).

[24] Y. Mairesse et al., Science 302, 1540 (2003).

[25] I.-L. Liu, P.-C. Li, and Shih-I Chu, Phys. Rev. A 84, 033414 (2011).

[26] P.-C. Li, I.-L. Liu, and Shih-I Chu, Opt. Express 19, 23857 (2011).

[27] X. M. Tong and S. I. Chu, Chem. Phys. 217, 119 (1997).

[28] M. B. Gaarde, J. L. Tate, and K. J. Schafer, J. Phys. B 41, 132001 (2008).

[29] C. Jin, A. T. Le, C. A. Trallero-Herrero, and C. D. Lin, Phys. Rev. A 84, 043411 (2011).

[30] M. Geissler, G. Tempea, A. Scrinzi, M. Schnürer, F. Krausz, and T. Brabec, Phys. Rev. Lett. 83, 2930 (1999).

[31] V. Tosa, H. T. Kim, I. J. Kim, and C. H. Nam, Phys. Rev. A 71, 063807 (2005).

[32] Ph. Antoine, A. L'Huillier, and M. Lewenstein, Phys. Rev. Lett. 77, 1234 (1996).

[33] X. M. Tong and Shih-I. Chu, Phys. Rev. A 61, 021802 (2000).

[34] A. L'Huillier, Ph. Balcou, S. Candel, K. J. Schafer, and K. C. Kulander, Phys. Rev. A 46, 2778 (1992).

[35] V. Tosa, K. T. Kim, and C. H. Nam, Phys. Rev. A 79, 043828 (2009). 